\title{
Music, Empathy, and Affiliation: Commentary on Greenberg, Rentfrow, and Baron-Cohen
}

\author{
JONNA K. VUOSKOSKI \\ University of Oxford
}

\begin{abstract}
Empathy and social cognition arguably play a crucial role in our engagement with music. In response to the account offered by Greenberg, Rentfrow, and Baron-Cohen, this commentary considers an alternative-yet complementaryexplanation for how music making and music listening might be able to evoke empathy and affiliation. This alternative explanation stems from the perception-action model of empathy, and the affiliation-evoking effects of mimicking and synchronized actions. In light of this alternative account, I will also explore the potential contribution of dispositional empathy to music preferences and music perception as suggested by Greenberg and colleagues.
\end{abstract}

Submitted 2014 October 10; accepted 2014 November 2.

KEYWORDS: empathy, affiliation, music listening, emotion, musical preference

GREENBERG, Rentfrow, and Baron-Cohen provide an intriguing account of the EmpathizingSystemizing theory in the context of music. In particular, they discuss the role of empathizing-a disposition to seek to understand others' feelings and to respond to them with appropriate emotion (e.g. Baron-Cohen \& Wheelwright, 2004) - in musical behaviors such as music listening and musical performance. Greenberg and colleagues outline how the Empathizing-Systemizing theory could be used to explain certain individual differences in music preferences as well as in the perception and performance of music. Furthermore, they suggest that listening to certain types of music might even be able to promote empathy in listeners. While I agree with the authors that dispositional empathy may account for certain individual differences in listeners' responses to (and perception of) music, I would like to consider some alternative - yet complementary — explanations for why and how this might be the case.

First, however, there is need to outline some key concepts. While dispositional empathy-as measured by the Empathy Quotient in the Empathizing-Systemizing framework, for example- is related to how people typically behave and react across a range of different situations, an individual's response in a specific situation could be characterized as "state empathy." There are various factors that influence whether or not we experience empathy in a given situation (and how strong that empathy is), albeit people with high dispositional empathy are more likely to experience empathy in general. For example, we are more likely to experience empathy for people that we are somehow affiliated with, such as people we love or care about, or people that belong to the same group as us (e.g. Avenanti et al., 2010).

\section{HOW IS MUSIC ABLE TO EVOKE EMPATHY?}

It has been proposed that empathy and theory of mind (or "mind reading") arise from shared neural representations of perception and action, where the observation of another's state will activate the corresponding areas in the observer's brain (e.g. Preston \& de Waal, 2002). Empathy, in essence, is implemented by simulating the mental states of other people, facilitated by the mirror neuron system (Iacoboni, 2009). Indeed, people with high dispositional empathy have been found to exhibit stronger somatomotor responses to others' pain (Avenanti et al., 2008), and stronger activation of the auditory mirror system in response to action sounds (Gazzola et al., 2006). With regard to music, it has been proposed that listeners could respond to music as they would to the perceived emotional state of a conspecific, resonating with those auditory and gestural features that resemble vocal and motor expression of emotion (Molnar-Szakacs \& Overy, 2006; Livingstone \& Thompson, 2009). In line with this account, 
there is empirical evidence indicating that people with high dispositional empathy are also more susceptible to emotional contagion from music (Vuoskoski \& Eerola, 2012).

Highly empathic people are also more likely to engage in nonconscious mimicry of interaction partners (Chartrand \& Bargh, 1999) - a behavior that has been shown to reflect affiliation and liking (Yabar et al., 2006). Interestingly, engaging in explicit mimicry of another's actions has also been found to evoke affiliation, possibly by increasing self-other overlap and "kick-starting" the brain system that underlies motor resonance (Inzlicht et al., 2012). Similarly, synchronized activities such as tapping and walking in step with others have been found to increase expectations of cooperation (Wiltermuth \& Heath, 2009), and affiliation and compassion towards confederates (Hove \& Risen, 2009; Valdesolo \& DeSteno, 2011), suggesting that there may be something intrinsically empathy-evoking about being together in time. It may be that music-making - like other synchronized activities-functions like mimicking, stimulating motor resonance and self-other merging. Tarr, Launay, and Dunbar (2014) suggest that this self-other merging might be accompanied by endorphin release, and that self-other merging and motor resonance could also take place during passive music listening.

These mechanisms could explain how music might be able to create and strengthen social bondsand evoke "state empathy." However, empirical evidence on the matter is only just starting to emerge. In a recent study, Vuoskoski, Clarke, and DeNora (in preparation) investigated whether listening to music from a particular culture could evoke empathy and increase affiliation towards members of that culture more generally. Manipulations that put us into the shoes of out-group members (figuratively speaking) - such as explicitly mimicking their actions (Inzlicht et al., 2012) - have previously been found to increase empathy and affiliation towards out-group members, and it was hypothesized that music listening might function in a similar way. Sixty-two participants listened to either Indian or West African popular music (with nonEnglish lyrics), and afterwards completed an Implicit Association Test measuring implicit affiliation with Indian relative to West African people. Vuoskoski and colleagues found a significant interaction of dispositional empathy and the type of music listened to: participants with high dispositional empathy scores were more likely to display an unconscious preference for the ethnic group to whose music they were exposed than those with low dispositional empathy scores. The fact that high dispositional empathy made participants more susceptible to the musical manipulations suggests that the observed findings cannot be explained in terms of priming or knowledge activation effects. Furthermore, the observed effects were not related to differences in liking for the musical pieces. Thus, this study provides preliminary empirical evidence that passive music listening could be able to evoke affiliation — potentially through affective and motor resonance and self-other merging.

While this account relates more specifically to state empathy, the explanation offered by Greenberg and colleagues could be interpreted as referring to dispositional empathy. They propose that listening to "mellow" music (i.e. music featuring romantic, relaxing, non-aggressive, sad, slow, and quiet attributes) might promote empathizing by facilitating reflective functioning and mentalizing abilities. This may indeed be the case, but would arguably require recurrent listening of such music. There is certainly evidence that long-term repeated participation in musical group interaction can increase emotional empathy (Rabinowitch et al., 2013), but empirical evidence regarding long-term music listening is currently lacking. Furthermore, it is unclear whether there might be something particularly empathy-evoking about mellow music, or whether "groovy" music (or other kinds of music that are likely to induce motor entrainment; see e.g. Janata et al., 2012), for example, could also evoke empathy to a similar or higher degree.

\section{EMPATHY AND MUSIC PREFERENCES}

Greenberg and colleagues propose that empathic people might be especially drawn to music that is "mellow," as this type of music may reflect the emotional depth that empathic people experience in their daily lives. Indeed, there is some empirical evidence indicating that people with high dispositional empathy prefer music that expresses sadness and tenderness (rather than music that expresses anger, fear, or happiness; Vuoskoski et al., 2012), but there might be an alternative explanation for the connection. As Greenberg and colleagues state, people typically prefer and seek out music that reflects and reinforces their personality characteristics (e.g. Rentfrow \& Gosling, 2003; Rentfrow et al., 2011). As empathic people have a stronger tendency to empathize with those undergoing negative emotions and to experience sympathy and concern (e.g., Davis, 1980), the same might hold true for music as well. In other words, empathic people may be more open to engage and empathize with sad-sounding music, and more likely to resonate with music expressing warm, compassionate feelings such as tenderness. It may also be that 
empathic people find it intrinsically pleasurable to engage in vicarious experiences, especially in aesthetic contexts. Dispositional empathy has been previously associated with both the susceptibility to musicinduced sadness (Vuoskoski \& Eerola, 2012) and the enjoyment of sad-sounding music (Vuoskoski et al., 2012), suggesting that there might be a link between empathically experienced emotions and enjoyment. It may be that empathic people are particularly sensitive to the effects of certain types of music, or that they only find certain music-induced emotions pleasurable.

\section{EMPATHY AND MUSIC PERCEPTION}

Finally, Greenberg and colleagues postulate that empathizing and systemizing might be related to differences in music perception - rhythm perception in particular. They suggest that empathizers might focus more on the emotional content of music, while systemizers-because of their enhanced attention to detail - would tend to focus more on sonic attributes such as rhythm. While systemizers and people with autism spectrum conditions might indeed pay more attention to detail and the "surface level" rather than on the "big picture," I find the idea of empathizers and systemizers differing in terms of rhythm and beat perception somewhat problematic. If the small sex difference in detection accuracy of off-beat metronome beeps observed by Müllensiefen et al. (2014) does indeed reflect differences in empathizing and systemizing, this difference may not be related to beat perception per se, but to differences in discrimination. It may be that empathizers are more "accommodating" in their grouping of rhythmic events, while systemizers capitalize on their greater attention to detail. However, empirical investigation is obviously required to explore this. I would like to suggest that beat perception actually plays an important role in listeners' empathic responses to music. First, beat perception is crucial for entrainment and synchronized activities, and thus should underlie self-other merging and the affiliation-evoking effects of music. Second, the same genetic markers (of the arginine vasopressin receptor 1A) are related to musical aptitude (including time discrimination) as well as social bonding and altruism (Ukkola et al., 2009). While rigorous empirical investigation is required to explore many of the hypotheses proposed by Greenberg and colleagues (and those presented in this commentary), it seems apparent that empathy and social cognition play a pivotal role in our engagement with music. Indeed, I hope that this new and exciting field of research will continue to gain momentum and attract significant research interest in the coming years, and advance our understanding of the intriguing relationship between music and empathy.

\section{REFERENCES}

Avenanti, A., Minio-Paluello, I., Bufalari, I., \& Aglioti, S. M. (2008). The pain of a model in the personality of an onlooker: Influence of state-reactivity and personality traits on embodied empathy for pain. Neuroimage, 44(1), 275-283.

Avenanti, A., Sirigu, A., \& Aglioti, S. M. (2010). Racial bias reduces empathic sensorimotor resonance with other-race pain. Current Biology, 20(11), 1018-1022.

Baron-Cohen, S., \& Wheelwright, S. (2004). The empathy quotient: An investigation of adults with asperger syndrome or high functioning autism, and normal sex differences. Journal of Autism and Developmental Disorders, 34(2), 163-175.

Chartrand, T. L., \& Bargh, J. A. (1999). The chameleon effect: The perception-behaviour link and social interaction. Journal of Personality and Social Psychology, 76(6), 893-910.

Davis, M. H. (1980). A multidimensional approach to individual differences in empathy. JSAS Catalog of Selected Documents in Psychology, 10, 85.

Gazzola, V., Aziz-Zadeh, L., \& Keysers, C. (2006). Empathy and the somatotopic auditory mirror system in humans. Current Biology, 16(18), 1824-1829.

Hove, M. J., \& Risen, J. L. (2009). It's all in the timing: Interpersonal synchrony increases affiliation. Social Cognition, 27(6), 949-960. 
Iacoboni, M. (2009). Imitation, empathy, and mirror neurons. Annual Review of Psychology, 60, 653-670.

Inzlicht, M., Gutsell, J. N., \& Legault, L. (2012). Mimicry reduces prejudice. Journal of Experimental Social Psychology, 48, 361-365.

Janata, P., Tomic, S. T., \& Haberman, J. M. (2012). Sensorimotor coupling in music and the psychology of the groove. Journal of Experimental Psychology: General, 141(1), 54-75.

Livingstone, R. S., \& Thompson, W. F. (2009). The emergence of music from the Theory of Mind. Musicae Scientiae, 13(2 suppl), 83-115.

Molnar-Szakacs, I., \& Overy, K. (2006). Music and mirror neurons: From motion to 'e'motion. Social Cognitive and Affective Neuroscience, 1(3), 235-241.

Müllensiefen, D., Gingras, B., Musil, J., \& Stewart, L. (2014). The musicality of non-musicians: An index for assessing musical sophistication in the general population. PloS One, 9(2), e89642.

Preston, S. D., \& De Waal, F. (2002). Empathy: Its ultimate and proximate bases. Behavioral and Brain Sciences, 25(1), 1-20.

Rabinowitch, T. -C., Cross, I., \& Burnard, P. (2013). Long-term musical group interaction has a positive influence on empathy in children. Psychology of Music, 41(4), 484-498.

Rentfrow, P. J., Goldberg, L. R., \& Levitin, D. J. (2011). The structure of musical preferences: A fivefactor model. Journal of Personality and Social Psychology, 100(6), 1139-1157.

Rentfrow, P. J., \& Gosling, S. D. (2003). The do re mi's of everyday life: The structure and personality correlates of music preferences. Journal of Personality and Social Psychology, 84, 1236-1256.

Tarr, B., Launay, J., \& Dunbar, R. I. (2014). Music and social bonding: "Self-Other" merging and neurohormonal mechanisms. Frontiers in Psychology: Auditory Cognitive Neuroscience, 5, 1096.

Ukkola, L. T., Onkamo, P., Raijas, P., Karma, K., \& Järvelä, I. (2009). Musical aptitude is associated with AVPR1A-haplotypes. PLoS One, 4(5), e5534.

Valdesolo, P., \& DeSteno, D. (2011). Synchrony and the social tuning of compassion. Emotion, 11(2), 262266.

Vuoskoski, J. K., Clarke, E. F., \& DeNora, T. (in preparation). Music listening evokes implicit affiliation. Manuscript in preparation.

Vuoskoski, J. K., \& Eerola, T. (2012). Can sad music really make you sad? Indirect measures of affective states induced by music and autobiographical memories. Psychology of Aesthetics, Creativity, and the Arts, 6(3), 204-213.

Vuoskoski, J. K., Thompson, W. F., McIlwain, D., \& Eerola, T. (2012). Who enjoys listening to sad music and why? Music Perception, 29(3), 311-317.

Wiltermuth, S. S., \& Heath, C. (2009). Synchrony and cooperation. Psychological Science, 20(1), 1-5.

Yabar, Y., Johnston, L., Miles, L., \& Peace, V. (2006). Implicit behavioral mimicry: Investigating the impact of group membership. Journal of Nonverbal Behavior, 30(3), 97-113. 